\section{(1)}

CrossMark

\title{
Genomic characterisation of pulmonary subsolid nodules: mutational landscape and radiological features
}

\author{
Yanmeng $\mathrm{Li}^{1,6}$, Xiao $\mathrm{Li}^{1,6}$, Hao Li $\mathbb{1}^{1,6}$, Yifan Zhao ${ }^{1,6}$, Ziyang Liu (1) ${ }^{1,6}$, \\ Kunkun Sun ${ }^{2}$, Xiang Zhu ${ }^{3}$, Qingyi Qi ${ }^{4}$, Bei $\mathrm{An}^{4}$, Danhua Shen ${ }^{2}$, Ruoyan $\mathrm{Li}^{1}$, \\ Taorui Liu ${ }^{1}$, Jiahui Mi $\mathbb{1}^{1}$, Lientu Wang ${ }^{5}$, Fan Yang ${ }^{1,7}$, Fan Bai ${ }^{1,7}$ and Jun Wang ${ }^{1,7}$ \\ Affiliations: 'Biomedical Pioneering Innovation Center (BIOPIC), School of Life Sciences and Dept of Thoracic \\ Surgery, People's Hospital, Peking University, Beijing, China. ${ }^{2}$ Dept of Pathology, Peking University People's \\ Hospital, Beijing, China. ${ }^{3}$ Dept of Pathology, Peking University Third Hospital, Beijing, China. ${ }^{4}$ Dept of \\ Radiology, Peking University People's Hospital, Beijing, China. ${ }^{5}$ Berry Oncology Co., Ltd, Fujian, China. ${ }^{6}$ These \\ authors contributed equally to the study. ${ }^{7}$ These authors contributed equally to the study.
}

Correspondence: Jun Wang, Dept of Thoracic Surgery, Peking University People's Hospital, No. 11 Xizhimen South Street, Beijing, 100044, China. E-mail: wangjunapkuph.edu.cn

@ERSpublications

This study provides the first comprehensive description of the mutational landscape of SSNs and reveals the potential genomic grounds supporting the use of radiological parameters as clinical references for diagnosis and treatment of SSNs http://bit.ly/2NDmV31

Cite this article as: $\mathrm{Li} \mathrm{Y}, \mathrm{Li} \mathrm{X}, \mathrm{Li} \mathrm{H}$, et al. Genomic characterisation of pulmonary subsolid nodules: mutational landscape and radiological features. Eur Respir J 2020; 55: 1901409 [https://doi.org/10.1183/ 13993003.01409-2019].

\section{ABSTRACT}

Background: Lung adenocarcinomas (LUADs) that display radiologically as subsolid nodules (SSNs) exhibit more indolent biological behaviour than solid LUADs. SSNs, commonly encompassing pre-invasive and invasive yet early-stage adenocarcinomas, can be categorised as pure ground-glass nodules and partsolid nodules. The genomic characteristics of SSNs remain poorly understood.

Methods: We subjected 154 SSN samples from 120 treatment-naïve Chinese patients to whole-exome sequencing. Clinical parameters and radiological features of these SSNs were collected. The genomic landscape of SSNs and differences from that of advanced-stage LUADs were defined. In addition, we investigated the intratumour heterogeneity and clonal relationship of multifocal SSNs and conducted radiogenomic analysis to link imaging and molecular characteristics of SSNs. Fisher's exact and Wilcoxon rank sum tests were used in the statistical analysis.

Results: The median somatic mutation rate across the SSN cohort was 1.12 mutations per Mb. Mutations in EGFR were the most prominent and significant variation, followed by those in RBM10, TP53, STK11 and KRAS. The differences between SSNs and advanced-stage LUADs at a genomic level were unravelled. Branched evolution and remarkable genomic heterogeneity were demonstrated in SSNs. Although multicentric origin was predominant, we also detected early metastatic events among multifocal SSNs. Using radiogenomic analysis, we found that higher ratios of solid components in SSNs were accompanied by significantly higher mutation frequencies in EGFR, TP53, RBM10 and ARID1B, suggesting that these genes play roles in the progression of LUADs.

Conclusions: Our study provides the first comprehensive description of the mutational landscape and radiogenomic mapping of SSNs. 


\section{Introduction}

Early-stage lung adenocarcinomas (LUADs) can present as a spectrum of radiological appearances, ranging from pure ground-glass nodules (pGGNs) generally thought to be indolent, to more aggressive solid LUADs. Radiological subsolid nodules (SSNs) can be further categorised as pGGNs, which are defined as nodules manifesting as an area of hazy increased lung opacity with preservation of bronchial and vascular margins, and part-solid nodules (PSNs) comprised of both ground-glass and solid components [1]. A proportion of pGGNs eventually develop into PSNs. Pathologically, malignant SSNs encompass pre-invasive lesions (atypical adenomatous hyperplasias and adenocarcinomas in situ), minimally invasive adenocarcinomas and invasive adenocarcinomas.

To date, comprehensive studies describing the genomic landscape [2-5] and clonal architecture [6, 7] of LUAD have focused mainly on relatively late-stage tumours. Genomic features of the very early stage of LUAD [8], especially the subgroup that displays radiologically as SSNs [9], remain poorly understood. Furthermore, the diverse clinical trajectories of pGGNs and PSNs pose significant management challenges, and there have been limited predictive and prognostic biomarkers to risk-stratify patients. Radiological features of SSNs, such as the subclassification (pGGN or PSN) [10], the solid size [11] and the consolidation tumour ratio (CTR) [12], can serve as important clinical references for diagnosis and treatment of this disease. However, the potential genomic grounds supporting the use of radiological parameters as important clinical references for diagnosis and treatment of SSNs were not deciphered.

In this study, we performed whole-exome sequencing of 154 surgical SSN specimens from 120 patients. We defined the genomic characteristics of SSNs and delineated potential driver somatic mutations underlying the development and progression to advanced-stage LUAD. We also revealed the intratumour heterogeneity between ground-glass and solid regions of the same SSN $(n=11)$ and the clonal relationship among multifocal SSNs from the same patient $(n=26)$. Finally, we provided radiogenomic mapping of SSNs. Our findings shed light on the evolution of LUAD and provide valuable biological and clinical insights into SSNs.

\section{Methods}

\section{SSN cohort}

Pathologically confirmed malignant tumour samples from 154 SSNs along with paired controls from 120 treatment-naïve Chinese patients were subjected to whole-exome sequencing. The study design is summarised in figure $1 \mathrm{a}$ and $\mathrm{b}$, and detailed clinical features of the cohort are summarised in table 1 and supplementary table S1. Of note, 11 large PSN samples were divided into two to five regions for whole-exome sequencing according to the gross appearance of the resected tumour and the radiological characteristics (figure 1c, supplementary table S2). Pathological diagnoses were classified as atypical adenomatous hyperplasia, adenocarcinoma in situ, minimally invasive adenocarcinoma or invasive adenocarcinoma according to the 2015 World Health Organization classification system (figure 1d).

\section{Radiological evaluation}

The maximum diameters of whole SSNs and solid components on lung windows were measured (figure 2b). The CTR was calculated as the ratio of the maximum diameter of consolidation divided by the tumour size. Volumetric parameters, such as solid volume percentage, were obtained semi-automatically using the Lung VCAR workstation (GE Healthcare, Chicago, IL, USA). The SSN mass was calculated using the following equation: mass=volume $\times(($ mean $C T$ attenuation +1000$) \times 0.01)$. Image analysis details are included in the supplementary material.

\section{Whole-exome sequencing library preparation and data analysis}

Protocols for genomic DNA extraction and whole-exome sequencing library preparation are detailed in the supplementary material. We used the Genome Analysis Toolkit (GATK, version 3.8.0) to pre-process the whole-exome sequencing data, and MuTect (version 1.1.4) and Strelka (version 2.8.4) to call single nucleotide variants and insertions and deletions, respectively. Further details of bioinformatic analyses arre provided in the supplementary material. We used Fisher's exact test and Wilcoxon rank sum test for statistical analysis.

\section{Results}

\section{The mutational landscape of SSNs}

The median (range) somatic mutation rate across the SSN cohort $(n=154)$ was $1.12(0.03-6.87)$ mutations per $\mathrm{Mb}$, which is significantly lower than that of Chinese advanced-stage LUADs [5] (supplementary figure S1). The median sequencing depth per tumour was 217. The mutational landscape of SSNs is summarised in figure 3a. 12 significant mutated genes were identified by the MutSigCV algorithm. Notably, mutations in EGFR were the most prominent and significant variation (50\%), followed by mutations in RBM10 (16\%), TP53 (13\%), STK11 (8\%) and KRAS (5\%). Other frequent, but not 
a)
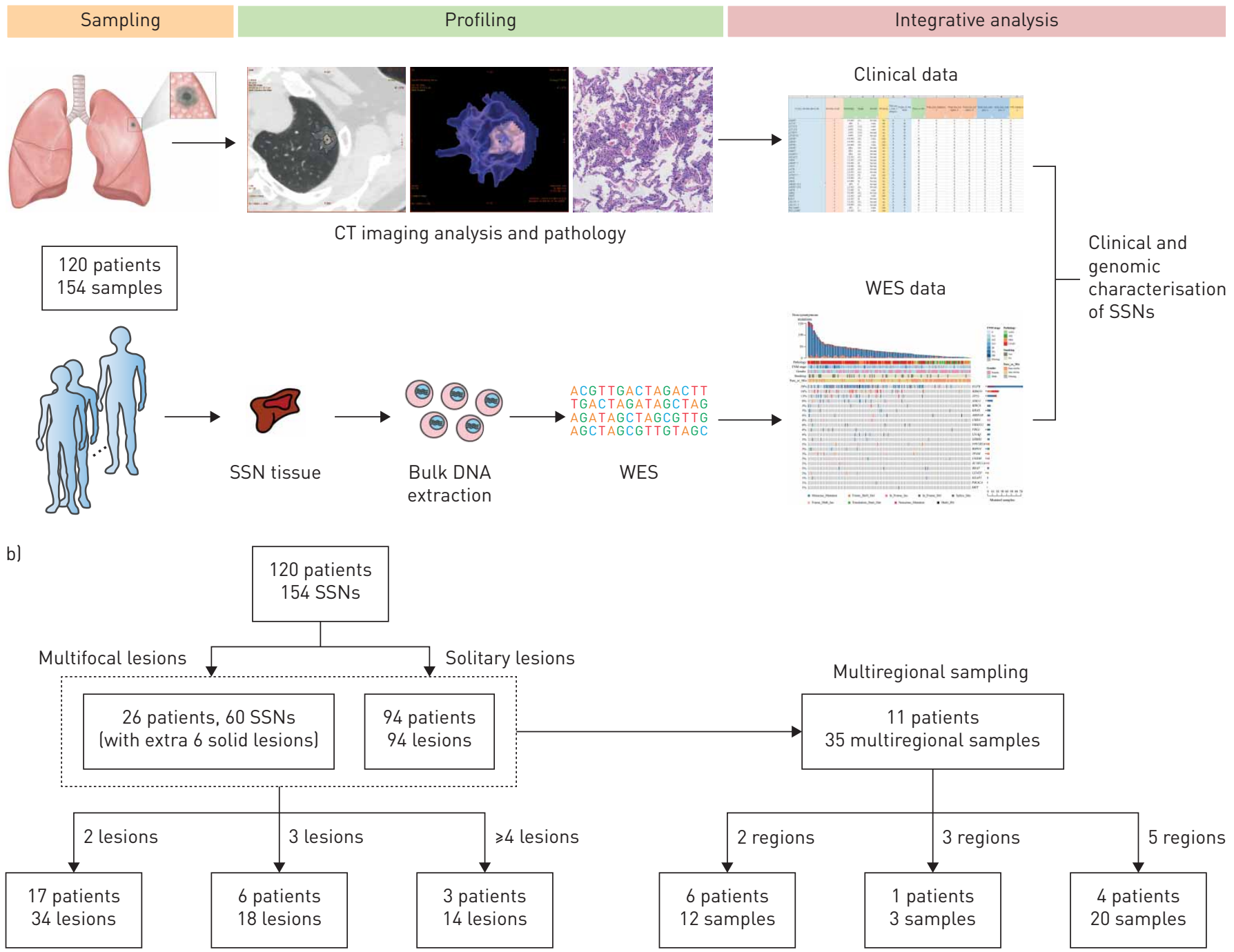

c)
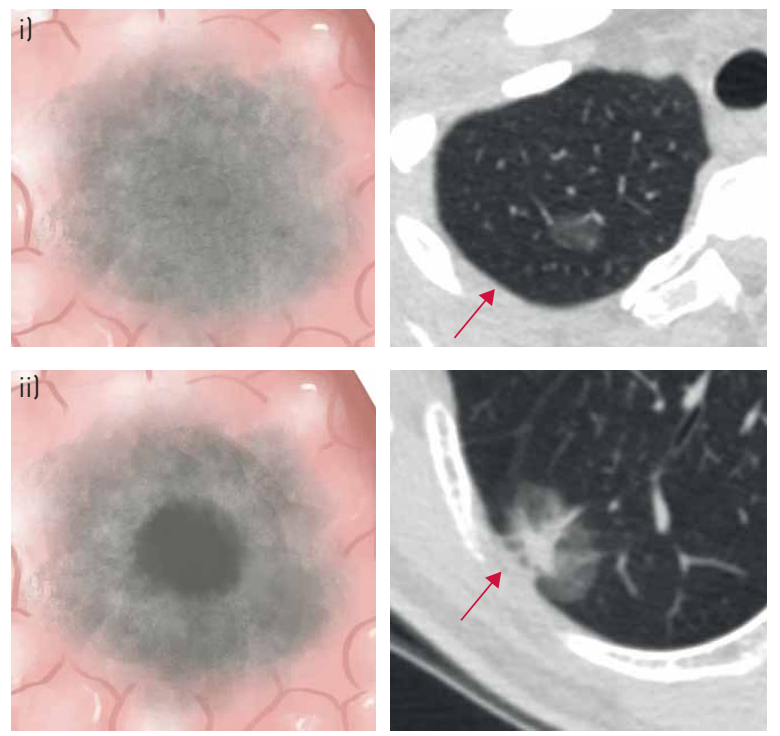

d)
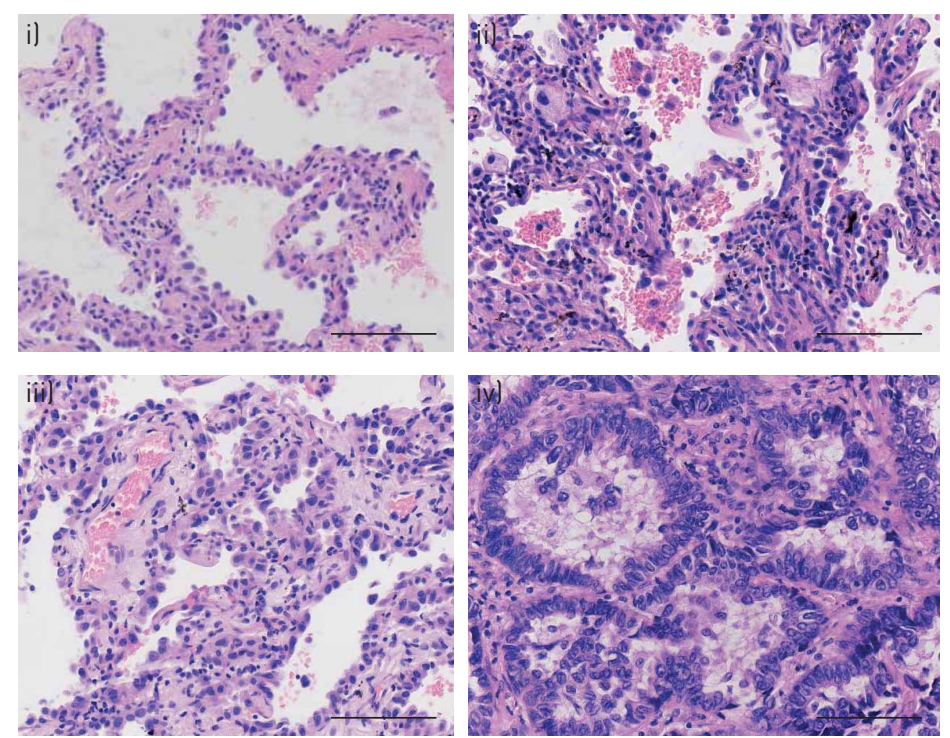
FIGURE 1 Study overview and sample information for 154 subsolid nodules (SSNs). a) Schematic of the study design; b) detailed information about the number of patients and samples assigned to different research strategies; c) schematic drawings of i) a pure ground-glass nodule and ii) a part-solid nodule with corresponding computed tomography (CT) images under the lung window setting (red arrows indicate sites of SSNs); d) histological features (haematoxylin and eosin staining) of SSNs including i) atypical adenomatous hyperplasia, ii) adenocarcinoma in situ, iii) minimally invasive adenocarcinoma iv) and invasive adenocarcinoma. WES: whole-exome sequencing. Scale bar=100 $\mu \mathrm{m}$.

significantly mutated genes included BIRC6 (5\%), ARID1B (4\%), CHD2 (4\%), FBXO11 (4\%) and GNAQ (4\%). Concurrent and mutually exclusive mutation patterns in SSNs are shown in figure 3d. Similar to previous reports in LUADs $[3,5,13]$, mutations in KRAS were mutually exclusive with those in EGFR. Both RBM10 and TP53 mutations commonly co-occurred with EGFR mutations. Interestingly, TP53 was found to be co-altered in only 19\% (15 out of 77) of the EGFR mutant population, lower than the

\section{TABLE 1 Clinical characteristics of 120 patients and 154 subsolid nodules (SSNs)}

\begin{tabular}{|c|c|}
\hline \multirow{2}{*}{\multicolumn{2}{|c|}{$\begin{array}{l}\text { Age years } \\
\text { Sex }\end{array}$}} \\
\hline & \\
\hline Female & $68(56.7)$ \\
\hline Male & 52 (43.3) \\
\hline \multicolumn{2}{|c|}{ Smoking status } \\
\hline Smoker & 32 (26.7) \\
\hline Nonsmoker & 88 (73.3) \\
\hline \multicolumn{2}{|c|}{ Lesion maximum diameter $\mathrm{mm}$} \\
\hline$\leqslant 10.0$ & $41(26.6)$ \\
\hline $10.1-20.0$ & $68(44.2)$ \\
\hline$>20.0$ & $45(29.2)$ \\
\hline \multicolumn{2}{|l|}{ SSN type } \\
\hline pGGN & 66 (42.9) \\
\hline PSN & 87 (56.5) \\
\hline NA & $1(0.6)$ \\
\hline \multicolumn{2}{|c|}{ Solid maximum diameter mm } \\
\hline$\leqslant 5$ & 79 (51.3) \\
\hline$>5$ & $74(48.1)$ \\
\hline NA & $1(0.6)$ \\
\hline \multicolumn{2}{|l|}{ CTR } \\
\hline $0-0.25$ & $68(44.2)$ \\
\hline$>0.25$ & 85 (55.2) \\
\hline NA & $1(0.6)$ \\
\hline \multicolumn{2}{|c|}{ Solid volume percentage \% } \\
\hline$\leqslant 50$ & 120 (77.9) \\
\hline$>50$ & $22(14.3)$ \\
\hline NA & $12(7.8)$ \\
\hline \multicolumn{2}{|l|}{ SSN mass mg } \\
\hline$\leqslant 250$ & 53 (34.4) \\
\hline$>250$ & 89 (57.8) \\
\hline NA & $12(7.8)$ \\
\hline \multicolumn{2}{|c|}{ Histological type } \\
\hline $\mathrm{AAH}$ & $14(9.1)$ \\
\hline AIS & $19(12.3)$ \\
\hline MIA & 30 (19.5) \\
\hline IAC & $91(59.1)$ \\
\hline \multicolumn{2}{|l|}{ Tumour stage $\mathrm{e}^{\#}$} \\
\hline NA $(\mathrm{AAH})$ & $14(9.1)$ \\
\hline 0 & 19 (12.3) \\
\hline$|\mathrm{A}|$ & $74(48.1)$ \\
\hline IA2 & $33(21.4)$ \\
\hline IA3 & $3(1.9)$ \\
\hline IB & $8(5.2)$ \\
\hline IIA & $1(0.6)$ \\
\hline IIB & $2(1.3)$ \\
\hline
\end{tabular}

Data are presented as median (range) or $\mathrm{n}(\%)$. pGGN: pure ground glass nodule; PSN: part-solid nodule; NA: not applicable; CTR: consolidation tumour ratio; AAH: atypical adenomatous hyperplasia; AIS: adenocarcinoma in situ; MIA: minimally invasive adenocarcinoma; IAC: invasive adenocarcinoma. \#: according to the International Union Against Cancer eighth tumour, node, metastasis staging. 

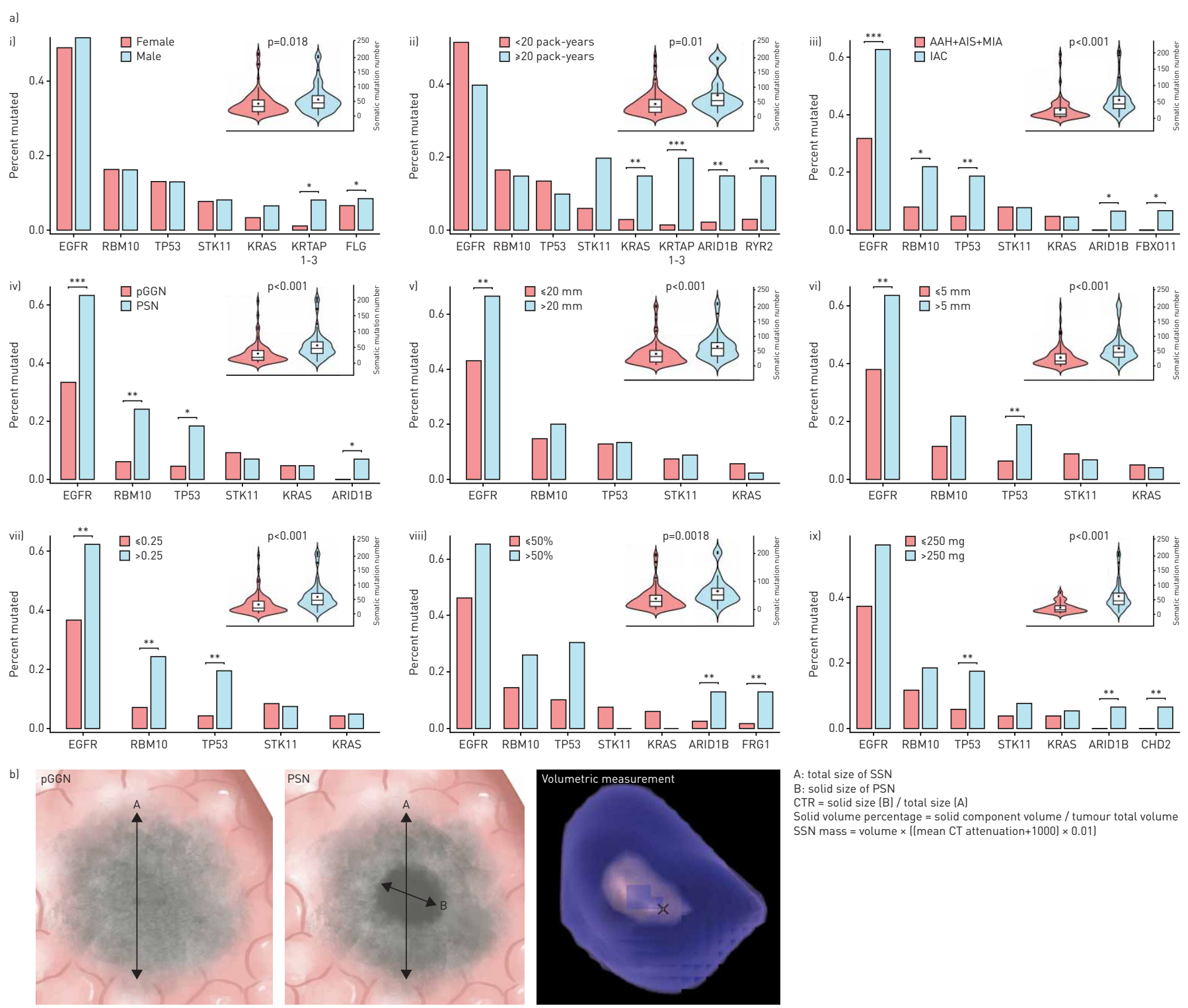

A: total size of SSN

B: solid size of PSN

$\mathrm{CTR}=$ solid size $(\mathrm{B}) /$ total size $(\mathrm{A})$

Solid volume percentage $=$ solid component volume $/$ tumour total volume $\mathrm{SSN}$ mass $=$ volume $\times(($ mean $\mathrm{CT}$ attenuation +1000$) \times 0.01)$

FIGURE 2 Clinical enrichment analysis of mutated genes by different clinical criterion groups. a) Both barplots, which indicate the mutation frequencies of significantly mutated genes as well as additional non-significantly enriched genes (for all genes with mutation frequencies $>3 \%$ ), and violin plots, which show the somatic mutation number, demonstrate the comparisons between sample groups classified by i) sex, ii) smoking condition with a cut-off of 20 pack-years, iii) pathology, iv) radiological subsolid nodule (SSN) classification (pure ground-glass nodule (pGGN) or part-solid nodule (PSN)), v) total size with a cut-off of $20 \mathrm{~mm}$, vil solid component size with a cut-off of $5 \mathrm{~mm}$, vii) consolidation tumour ratio (CTR) with a cut-off of 0.25 , vii) solid volume percentage with a cut-off of $50 \%$ and viii) SSN mass with a cut-off of $250 \mathrm{mg}$. AAH: atypical adenomatous hyperplasia; AIS: adenocarcinoma in situ; MIA: minimally invasive adenocarcinoma; IAC: invasive adenocarcinoma. For the barplots, significance was calculated by Fisher's exact test; for the violin plots, the $p$-value was calculated by Wilcoxon rank sum test. *: $p<0.05,{ }^{* *}$ : $p<0.01$, ***: $p<0.001$. b) Schematic diagrams and formulas for calculation of several of the clinical criteria mentioned in (a).

anticipated $50-60 \%$ in early-stage solid tumours or metastatic disease $[5,14]$, suggesting that these combinations may facilitate the transformation of early stage lung neoplasm to malignancy [15].

We further explored the aetiological factors that underlie mutagenesis in Chinese SSNs. Mutational spectrum analysis revealed a strong enrichment of $\mathrm{C}>\mathrm{T}$ transversions and $\mathrm{C}>\mathrm{A}$ transitions, which are associated with a history of smoking (figure 3b). From 154 SSNs, we confidently identified three mutational signatures, which displayed high similarity to COSMIC signatures 4, 6 and 5 (figure 3c) [16]. COSMIC signature 4 is associated with smoking and has been frequently reported in LUADs $[4,5]$. Intriguingly, COSMIC signature 6, which is often identified in colorectal cancers but less in LUADs [2], is characteristic of tumours with defective DNA mismatch repair. These results suggested that smoking and dysregulation of DNA repair contribute to SSN tumourigenesis. 
Comparing the genomic features of SSNs to advanced-stage LUADs

To unravel the differences between SSNs and advanced-stage LUADs at a genomic level, we compared the mutation frequencies of driver genes in 10 oncogenic pathways [17] and other previously reported mutations related to $\operatorname{LUAD}[3,5]$. The mutation frequencies of these genes in advanced-stage LUADs were calculated by reanalysing data from the Cancer Genome Atlas (106 samples of stage II-IV LUADs [3]) and published Chinese LUADs (75 samples of stage II-IV LUADs [5]) (figure 3e, supplementary figure S2).

First, we compared the mutation frequencies of oncogenic pathways in SSNs and stage II-IV LUADs and found the differences between the three groups (supplementary table S3). Next, we examined the frequencies of driver mutations in SSNs and Chinese stage II-IV LUADs (figure 3e, supplementary figure S2). The smoking status of these two cohorts were similar (73.3\% nonsmokers in Chinese SSNs and $61.3 \%$ in Chinese stage II-IV LUADs). Of note, EGFR (50.0\% versus $30.7 \%$ ) and RBM10 (16.2\% versus $1.3 \%$ ) mutations exhibited significantly higher frequencies in SSNs. Of all the 83 EGFR mutations from 77 SSNs, $60 \%$ ( 50 out of 83 ) were L858R, and 20\% (17 out of 83 ) were exon 19 deletions, while the remainder (16 out of 83) were other rare mutations (T790M, L858M, L861Q, L833F, and so on) (supplementary figure S3). This was consistent with a previous study which reported that EGFR mutations were found in $64 \%$ of Japanese SSNs [9]. Interestingly, RBM10 exhibited a significantly higher mutation frequency in SSNs with specific mutational types (nonsense, frameshift and splice site) (supplementary figure S3) [4] when compared to Chinese advanced-stage LUADs. RBM10, which encodes an RNA-binding protein, was found to be frequently mutated (7\%) in Caucasian LUADs [4] and highly mutated in pre-invasive and early-stage LUADs [18]. We speculated that alterations in RNA splicing alterations are a hallmark of SSNs, but do not confer a sustained evolutionary advantage during progression to advanced stage adenocarcinoma.

A number of genes displayed notably lower mutation frequencies in SSNs when compared with Chinese stage II-IV LUADs (figure 3e). These included tumour suppressor genes in key oncogenic pathways, including TP53, APC, FAT1/2/3/4, CRB1/2, NOTCH1/2/3/4, SPEN and ARID1A and oncogenes including KRAS, ERBB4, ROS1, PIK3CA/B, TEAD1/2 and NFE2L2, as well as genes associated with cytoskeleton remodelling including $P H P N 2$ and TRIO. Above all, we speculated that EGFR and RBM10 mutations are critical for SSN tumourigenesis, but progression of SSNs to advanced-stage LUADs is driven by mutations in other important cancer-related genes.

\section{Genomic profile of intrapatient multifocal SSNs}

Multiple synchronous occurrence is a feature of SSNs. To explore the clonal relationship between multifocal SSNs and solid tumours from the same patient, we sequenced 66 samples from 26 patients (two to five per patient). The interlesional genomic profile (the median sequencing depth of these regions was 186.9) was distinct in 23 out of 26 patients and generally confirmed the multicentric origin of SSNs (supplementary figure S4).

However, of note, P119 with four pGGNs (T1, T3-T5) and one PSN (T2) was found to have a metastatic event (between T4 and T5). P119-T4 and P119-T5, both of which were located in the apical-posterior segment of left upper lobe and histologically presented as minimally invasive adenocarcinomas, were demonstrated to share 10 nonsynonymous and eight synonymous mutations (figure $4 \mathrm{a}$ ). The potential driver MLLT1 mutation was shared by these two pGGNs, while EGFR(L858R), TP53 and PASK mutations were exclusive to P119-T4. Taken together, multicentric tumours and intrapulmonary metastatic lesions with a common clonal origin co-existed in this patient, which can be visualised in the regional distribution of mutations and phylogenetic tree.

We identified three PSNs (T1-T3) and one solid nodule (T4) of P114 that originated from the same clonal origin (figure $4 \mathrm{~b}$ ). P114-T1 showed predominant solid features by computed tomography scan (CTR 0.87 , solid volume percentage 68\%), mainly acinar in pathology. P114-T2 (CTR 0.39, solid volume percentage 30\%) and P114-T3 (CTR 0.66, solid volume percentage 41\%) were ground-glass-predominant lesions with adenocarcinoma in situ and mainly acinar in their pathology, respectively. P114-T1 was very large and therefore underwent multiregion sequencing (R1-R5). The phylogenetic tree of this patient revealed that P114-T1-R1 R5, P114-T2, P114-T3, and P114-T4 all shared a substantial amount of mutations. Interestingly, P114-T1 displayed remarkable regional heterogeneity and the most central part (R5) of P114-T1 was the nearest to the putative metastases (P114-T2, P114-T3 and P114-T4) in evolutionary distance, suggesting that metastatic lesions of SSNs can either stay as SSNs or progress to solid tumours.

\section{Intratumour heterogeneity between the ground-glass and solid components of SSNs}

To investigate the evolution and intratumour heterogeneity of SSNs, we performed multiregion sequencing on 35 spatially separate regions sampled from 11 large SSNs. The regional mutation distributions and 
a)

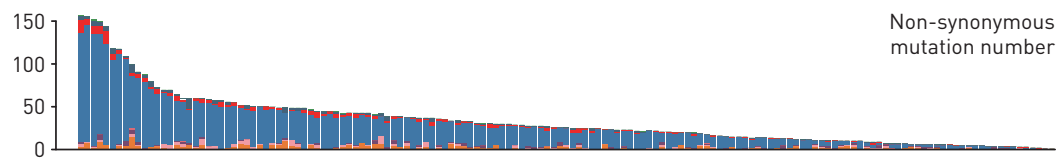

Pathology

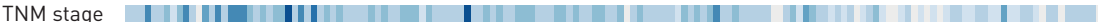
Sex

Smoking |

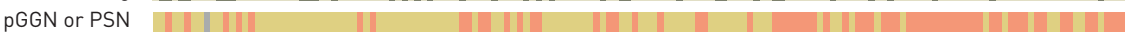

$\begin{array}{cc}\text { TNM stage } & \text { Pathology } \\ \text { AAH } & \text { AAH } \\ \text { OAH } & \text { AIS } \\ \text { IA1 } & \text { MIA } \\ \text { IA2 } & \text { IAC } \\ \text { IA3 } & \text { Smoking } \\ \text { IB } & \text { Yes } \\ \text { IIA } & \text { No } \\ \text { IIB } & \text { pGGN or PSN } \\ & \text { pGGN } \\ \text { Sex } & \text { PSN } \\ \text { Female } & \text { Missing } \\ \text { Male } & \end{array}$

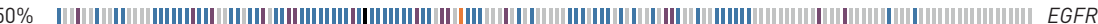

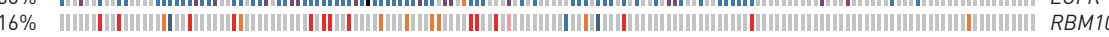

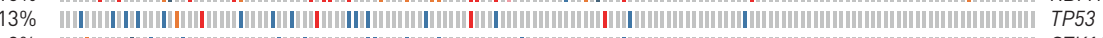

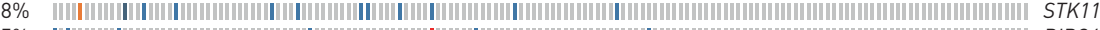
5\%

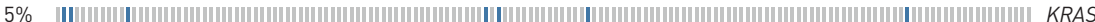
4\% 4\% 4\% 4\%

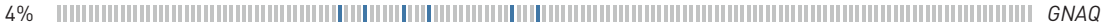
$3 \%$. 3\% 3\%

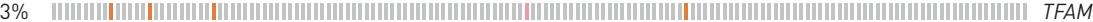
3\%

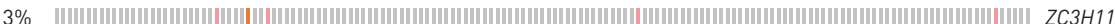

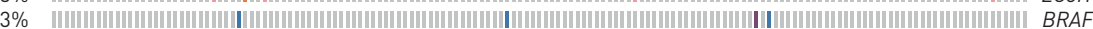
2\%

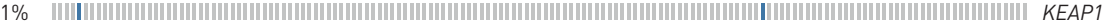

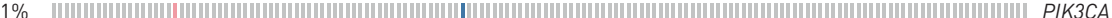

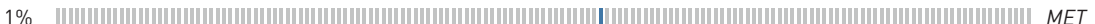
Missense mutation Frame shift del In frame ins In frame del Splice site

b)

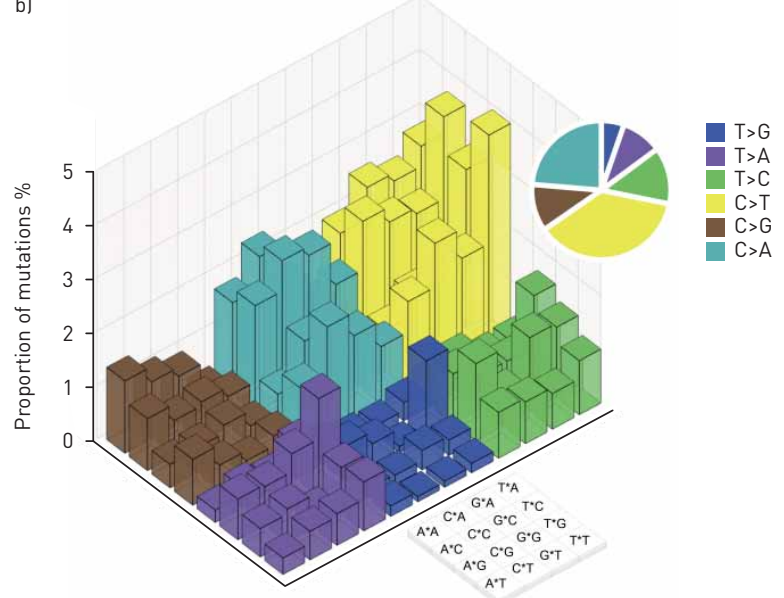

[* [* โ

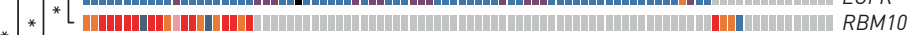

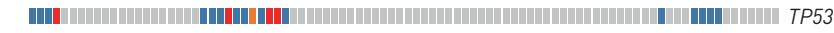

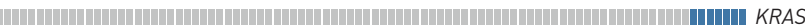
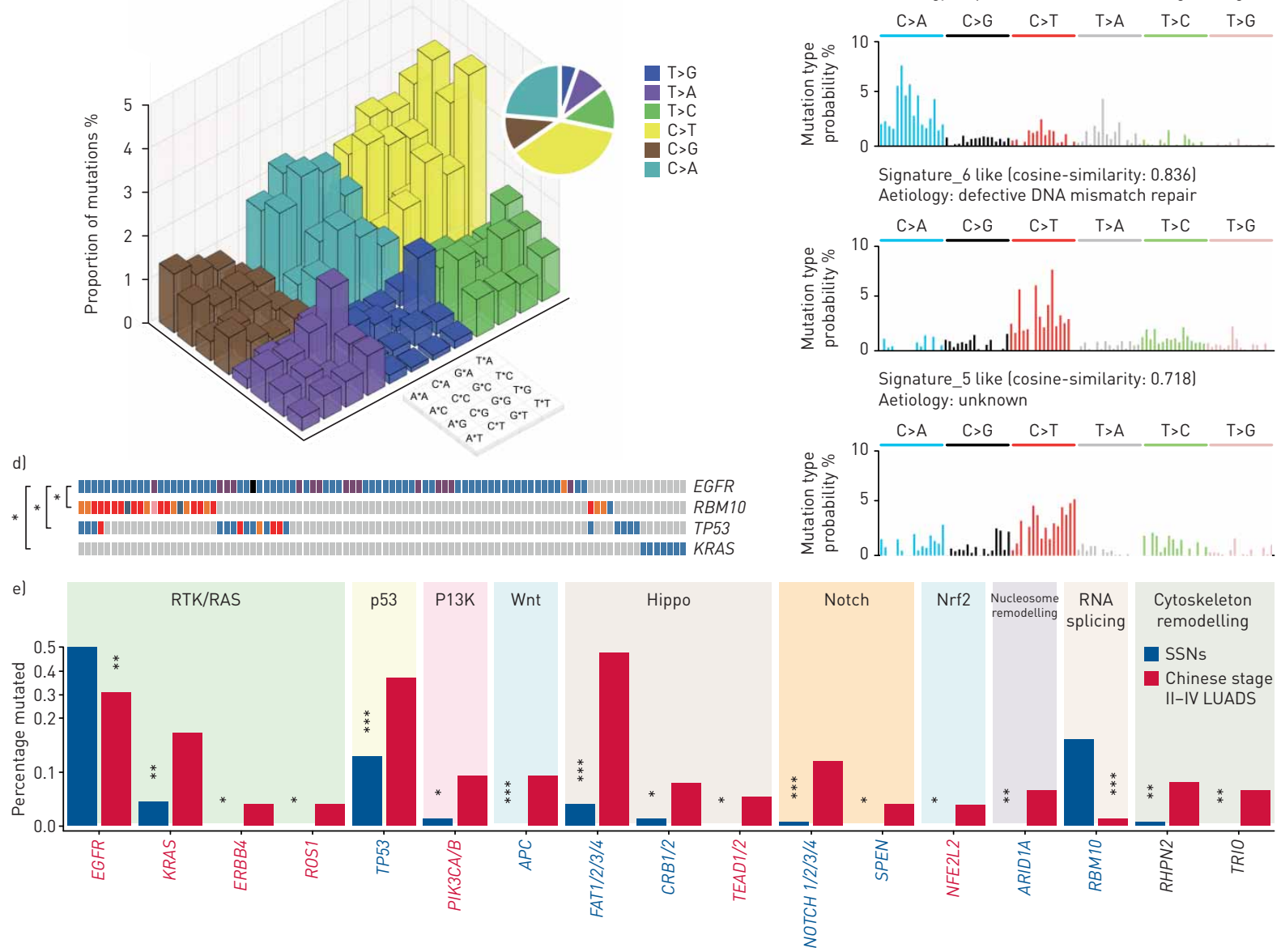

Signature_4 like (cosine-similarity: 0.925 )
Aetiology: exposure to tobacco (smoking) mutagens
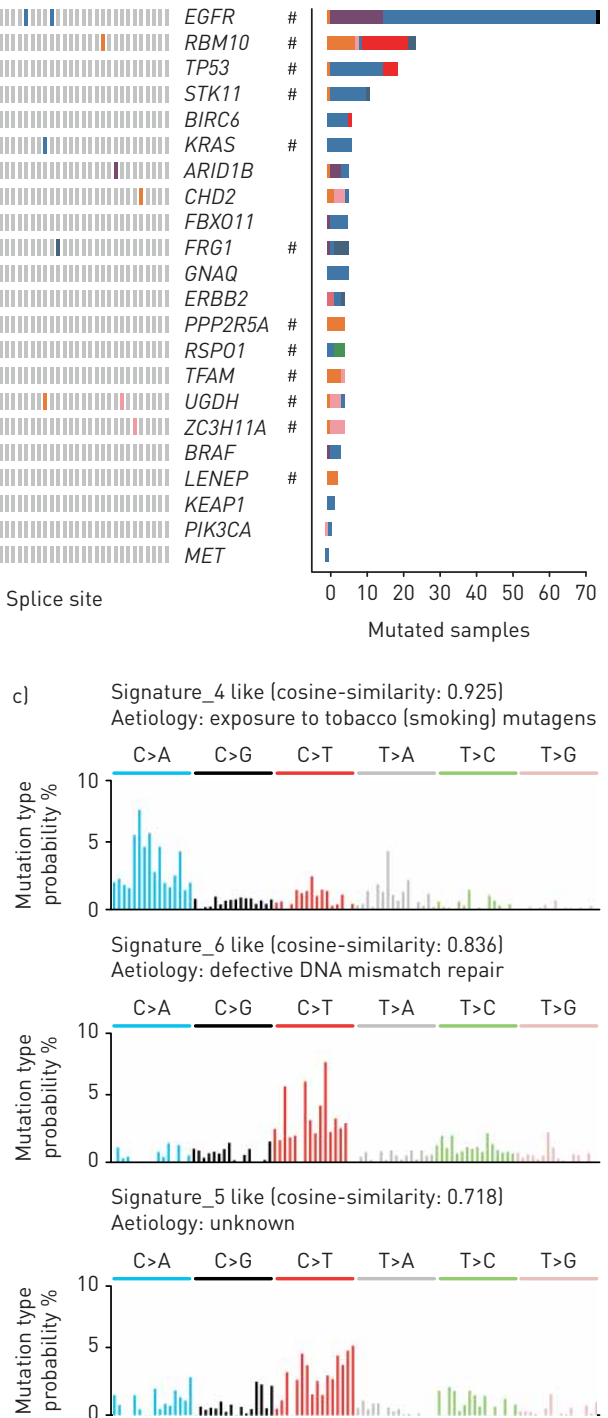
FIGURE 3 The mutational landscape and signatures of Chinese subsolid nodules (SSNs). a) Mutational landscape of nonsynonymous somatic mutations, including single nucleotide variants and insertions (ins) and deletions (del). Samples displayed as columns are ordered by the number of nonsynonymous somatic mutations, as shown in the upper panel. Clinical features are annotated in the middle panel. The heatmap below displays the somatic mutated genes ordered by their respective mutation frequencies. TNM: tumour, node, metastasis; pGGN: pure ground-glass nodule; PSN: part-solid nodule; AAH: atypical adenomatous hyperplasia; AIS: adenocarcinoma in situ; MIA: minimally invasive adenocarcinoma; IAC: invasive adenocarcinoma. ": 12 significant genes with a corrected q-value < 0.1 were identified by the MutSigCV algorithm. bl "Lego" plots displaying the frequency of base substitutions within specific trinucleotide mutational contexts. c) Mutational signatures of Chinese SSNs. d) Concurrent and mutually exclusive somatic mutation patterns of significantly mutated genes. Significance was calculated using Fisher's exact test. e) Comparison of the mutation frequencies of important genes in cancer-associated pathways between Chinese SSNs and Chinese advanced-stage lung adenocardinomas (LUADS; stage II-IV) [5]. Oncogenes labelled in red and tumour suppressor genes labelled in blue are listed on the horizontal axis ordered by the corresponding pathways. The vertical axis represents the mutation frequencies obtained from different cohorts. Significance was calculated using Fisher's exact test. *: $p<0.05,{ }^{* *}: p<0.01,{ }^{* * *}: p<0.001$.

phylogenetic trees of P118, P117 and P116 are shown in figure 4c, d and e, respectively (results for the remaining eight patients are included in supplementary figure S5). Branched evolution was evident in SSNs, with potential driver mutations presenting both on the trunks and branches in the phylogenetic trees (figure $4 \mathrm{c}, \mathrm{d}$ and e). This demonstrated that genomic heterogeneity and branched evolution are salient features of LUADs, even in their early stages when they radiologically display as SSNs.

Next, we focused on the difference between the ground-glass and solid components of PSNs in the hopes of uncovering genomic factors that shape the distinct radiological phenotypes within the same lesion. Interestingly, we found that solid components had comparable or even fewer somatic mutation numbers than the ground-glass components, and no significant enrichment of specific mutations was identified in ground-glass or solid components. The evolutionary distance between regions of the same radiological phenotype varied among patients, as demonstrated in the phylogenetic trees (figure $4 \mathrm{~b}, \mathrm{c}, \mathrm{d}$ and e). For example, R4 and R5 sampled from the solid component of P114-T1 were remarkably heterogeneous. The same result was observed among R1, R2 and R3, which were sampled from the ground-glass component of P114-T1 (figure 4b). In contrast, R2 and R3 from the ground-glass component of P117-T displayed high genomic similarity, whereas R1, R4 and R5 from the solid component grouped together on the phylogenetic tree (figure $4 \mathrm{~d}$ ), consistent with their separation in the radiological phenotype. In this regard, mutational features are not deterministically correlated with radiological features of different regions in the same SSN.

\section{Radiogenomic analysis links imaging and molecular characteristics of SSNs}

Radiological features of SSNs can serve as important clinical references for diagnosis and treatment. Given the clinical significance of these radiological parameters of SSNs, we investigated the differences in the number of somatic mutations and patterns of mutated genes (for all genes with mutation frequencies $>3 \%$ ) between any two groups separated by thresholds of each parameter with relevant clinical implications.

The mutation patterns were first correlated with some basic clinical features such as sex, smoking condition and pathology (figure $2 \mathrm{a}(\mathrm{i}$, ii and iii), respectively). Driver mutations of SSNs, including EGFR, RBM10, TP53 and STK11 displayed roughly the same mutation frequencies between sexes, an observation which differed from previous studies [3,5]. KRAS mutations were more commonly observed in males, although the difference was not statistically significant. In addition, we found that KTRAP1-3 mutations were significantly enriched in males, although FLG mutations were enriched in female patients $(\mathrm{p}<0.05$, Fisher's exact test). Compared with nonsmokers and light smokers ( $<20$ pack-years), medium-heavy smokers ( $\geqslant 20$ pack-years) had significantly greater numbers of KRAS, KTRAP1-3 and ARID1B mutations $(\mathrm{p}<0.01$ or $\mathrm{p}<0.001$, Fisher's exact test) along with a higher somatic mutation burden $(\mathrm{p}=0.01$, Wilcoxon rank sum test). When considering the pathological characteristics of the SSNs (supplementary table S4), the mutation frequencies of EGFR, RBM10, TP53, ARID1B and FBXO11 were significantly greater in SSNs that pathologically presented as invasive adenocarcinoma compared to those that presented as atypical adenomatous hyperplasia, adenocarcinoma in situ and minimally invasive adenocarcinoma $(\mathrm{p}<0.05$, Fisher's exact test). In addition, we found that invasive adenocarcinoma SSNs possessed a much higher somatic mutation load ( $\mathrm{p}<0.001$, Wilcoxon rank sum test).

We next investigated the relationship between the radiological predictors of invasiveness and the genomic profiles of SSNs. Similar to the results found for pathology, PSNs in our cohort were enriched for EGFR, RBM10, TP53 and ARID1B mutations ( $\mathrm{p}<0.05$, Fisher's exact test) and displayed significantly higher numbers of somatic mutations ( $\mathrm{p}<0.001$, Wilcoxon rank sum test) than did pGGNs (figure $2 a(i v)$ ). We then compared the patterns of mutation of the subgroups separated by total size and solid size (figure $2 \mathrm{a}(\mathrm{v}$ and vi)). Only mutation in EGFR was significantly enriched in SSNs with a total size $>20 \mathrm{~mm}$ when compared to smaller SSNs $(\mathrm{p}<0.05$, Fisher's exact test). However, when considering the size of the solid 
a)

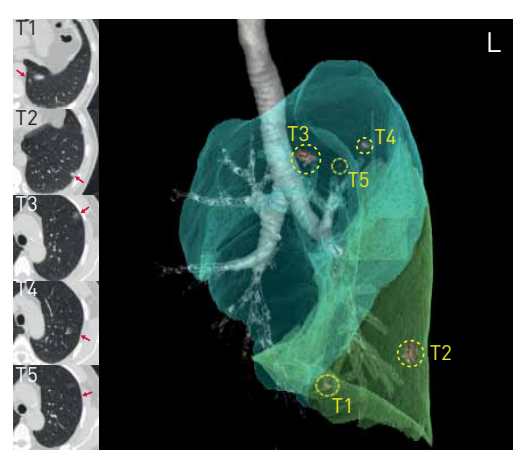

(11) 12 (13) (14) 15
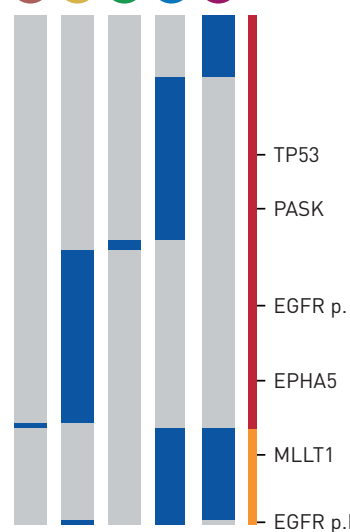

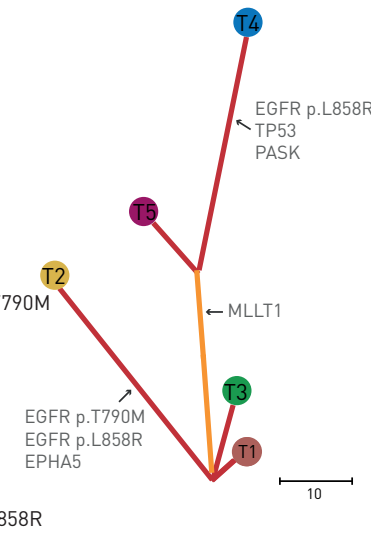

P117

d)

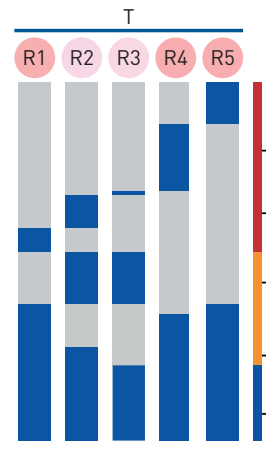

e)
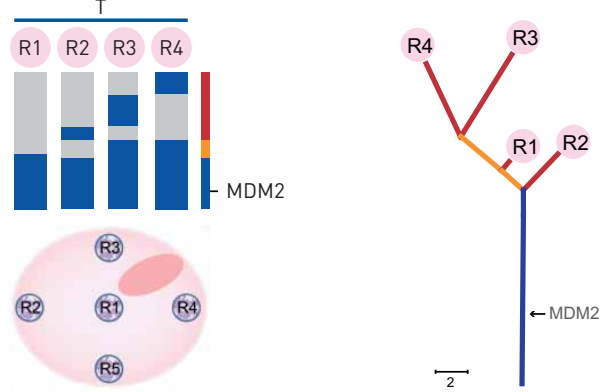

b)

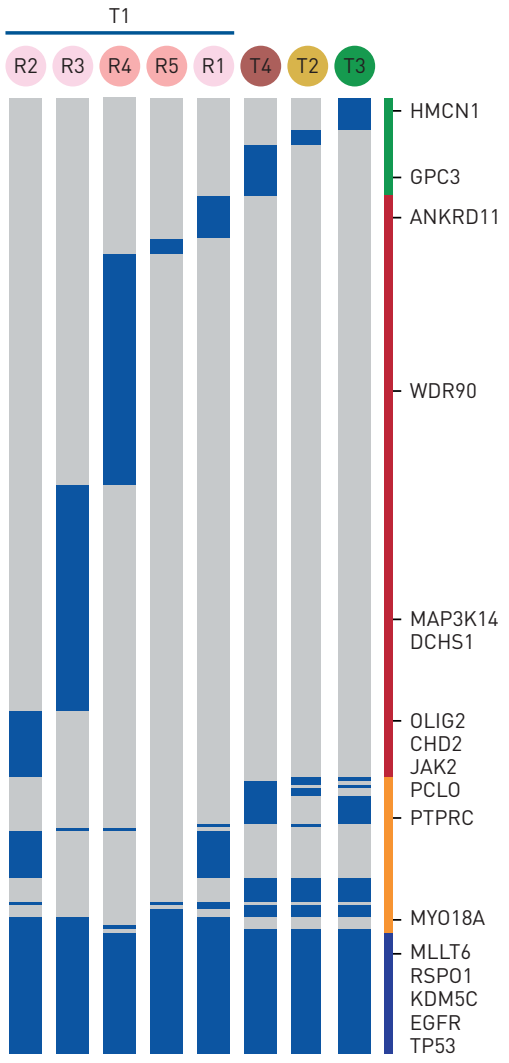

c)

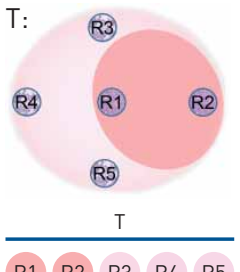

$\begin{array}{lllll}\text { R1 } & \text { R2 } & \text { R3 } & \text { R4 } & \text { R5 }\end{array}$

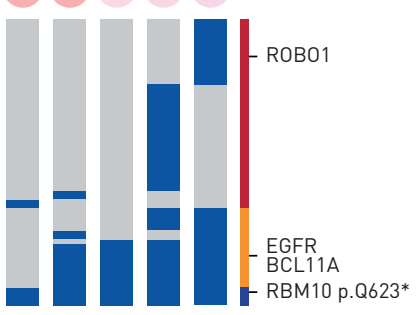

Mutation present

Mutation absent

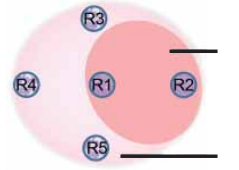

P114
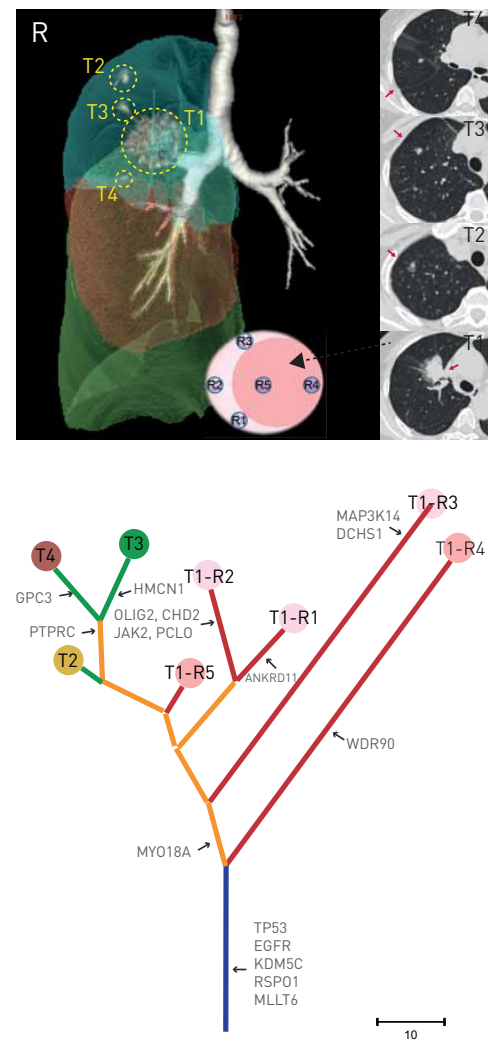

P118

- Trunk mutations

- Shared branch mutations

- Private branch mutations (multi regions in one SSN)

- Private branch mutations

lother tumors of the same patient)

Solid component of PSN

Ground-glass component of PSN

FIGURE 4 Intertumour and intratumour heterogeneity of subsolid nodules (SSNs). Heatmaps on the left of each panel depict the regional distribution of all of the somatic mutations among different sequencing sites of multiregion SSNs and multifocal SSNs. Phylogenetic trees on the right of each panel were constructed for each patient using the maximum parsimony algorithm. The colour of each line corresponds to the categories of mutations shown in the heatmaps, and the lengths of the trunks and branches are proportional to the number of mutations in each sample. Spatial location information of multifocal samples in b) P114 and a) P119 is represented in the three-dimensional reconstructions with the corresponding computed tomography images on the side. L: left lobe. R: right lobe. 
component, in addition to EGFR, TP53 was also significantly enriched in SSNs with a solid size $>5 \mathrm{~mm}$ $(\mathrm{p}<0.01$, Fisher's exact test). This finding underscored the importance of mutation of TP53 in driving the growth of the invasive tumour component (solid size) rather than the total SSN growth (total size). In contrast, mutation in EGFR may play an important role in both SSN growth and SSN invasiveness. Both groups (total size $>20 \mathrm{~mm}$ and solid size $>5 \mathrm{~mm}$ ) exhibited much higher somatic mutation burdens than other groups $(\mathrm{p}<0.01$, Wilcoxon rank sum test) (figure $2 \mathrm{a}(\mathrm{v}$ and $\mathrm{vi})$ ).

The CTR, solid volume percentage and SSN mass are additional predictive radiological parameters that are used to evaluate the tumour solid components (figure $2 \mathrm{a}$ (vii, viii and ix)). We found that the number of somatic mutations was significantly higher in groups with a CTR $>0.25$, a solid volume percentage $>50 \%$ and an SSN mass $>250 \mathrm{mg}$, which suggested that increased solid component in SSNs is correlated with increased mutation events. EGFR, RBM10 and TP53 mutations were significantly enriched in SSNs with a CTR $\geqslant 0.25$ ( $p<0.01$, Fisher's exact test). Similar enrichments were found when comparing subgroups classified by solid volume percentage and mass, although without statistical significance. However, these two classification groups both displayed a higher frequency of $A R I D 1 B$ mutations ( $\mathrm{p}<0.01$, Fisher's exact test).

\section{Discussion}

In this study, we provided a comprehensive genomic landscape of Chinese SSNs. We highlighted that mutations in genes including EGFR, RBM10, TP53, STK11, BIRC6 and KRAS play important roles in driving SSN tumourigenesis and discovered mutational signatures associated with smoking and defective DNA mismatch repair, the latter of which is often identified in colorectal cancer [16], but is rare in LUADs [19]. Consistent with this observation, genes associated with DNA repair, such as ATM [20], ASXL1 [21], ATRX [22] and ARID1B [23], were also mutated in SSNs as well as most early lesions of LUADs [15]. By comparing the genomic features of SSNs to those of advanced-stage LUADs, we were able to delineate the potential order of driver mutations during the progression of SSNs to invasive LUADs. These findings may explain why SSNs are able to remain indolent for many years.

Our study confirmed that most multifocal SSNs are tumours of multiple synchronous occurrence. However, we identified two cases with metastatic multifocal SSNs. Together with the two cases we reported previously [24], we found that SSNs can disseminate metastatic lesions while the metastatic lesions can remain as SSNs. The exact metastatic routes remain unknown, but are possibly through haematogenous, lymphatic or airway metastasis. We demonstrated that genomic heterogeneity and branched evolution are present even in the early stages of LUADs, as shown in a recent study [25], highlighting the complex evolutionary history of lung cancers and the therapeutic challenges we face.

It is widely accepted that radiological features of SSNs can serve as important clinical parameters for the diagnosis and treatment of SSNs. The total size and solid size of SSNs can predict their clinical behaviours. A more aggressive surveillance strategy should be applied to patients with pGGNs with a total size $>20 \mathrm{~mm}$ [26]. CTR, which takes both total size and solid size into account, also plays a critical role in the evaluation and treatment of SSNs [27]. Meanwhile, tumour volume and mass are novel indexes that reflect the invasiveness of SSNs [28]. Using radiogenomic analysis, we revealed the potential genomic grounds supporting the use of these parameters as important clinical references for diagnosis and treatment of SSNs. To summarise, when considering the total size of SSNs, only mutation in EGFR was remarkably different between subgroups stratified by this parameter. However, when we took the presence (pGGN or PSN), the size (solid size), and the weight (CTR, solid volume percentage, mass) of solid components into consideration, we found that more solid components were accompanied by significantly higher mutation frequencies in EGFR, TP53, RBM10 and ARID1B. We revealed that the formation of the solid components of SSNs is potentially driven by additional mutations in cancer-related genes. Together with the relationship between pathological characteristics and genomic profiles of SSNs, higher mutation frequencies in EGFR, TP53, RBM10 and $A R I D 1 B$ were proven to relate to a more invasive behaviour radiologically and pathologically.

Acknowledgements: We thank Weiwei Zhai (Institute of Zoology, Chinese Academy of Sciences, Beijing, China) for helpful discussions. We thank all the patients for their participation in this study.

Author contributions: F. Yang, F. Bai and J. Wang designed and supervised the study. Y. Zhao and Y. Li carried out the experiments. Y. Li, Z. Liu and R. Li analysed whole-exome sequencing and clinical data. Q. Qi and B. An performed the radiological analyses of subsolid nodules. K. Sun, X. Zhu and D. Shen performed the histological examination. T. Liu and J. Mi collected the clinical data. Y. Li compiled graphs and figures. Y. Li, X. Li, H. Li and F. Bai wrote the manuscript. All authors have read and agreed to the final version of the manuscript.

Support statement: This work was supported by the National Key Research and Development Program (grant 2016YFC0900102 to F. Bai); the National Science and Technology Major Project (grant 2018ZX10302205 to F. Bai); and the National Natural Science Foundation of China (grant 81772469 to F. Yang). The whole-exome sequencing data 
generated and analysed during the current study are available in the Genome Sequence Archive (Genomics, Proteomics and Bioinformatics 2017) in BIG Data Center (Nucleic Acids Res 2018), Beijing Institute of Genomics (BIG), Chinese Academy of Sciences, under accession numbers CRA001568 (publicly accessible at http://bigd.big.ac.cn/gsa). Funding information for this article has been deposited with the Crossref Funder Registry.

Conflict of interest: None declared.

\section{References}

1 Naidich DP, Bankier AA, MacMahon $\mathrm{H}$, et al. Recommendations for the management of subsolid pulmonary nodules detected at CT: a statement from the Fleischner Society. Radiology 2013; 266: 304-317.

2 Campbell JD, Alexandrov A, Kim J, et al. Distinct patterns of somatic genome alterations in lung adenocarcinomas and squamous cell carcinomas. Nat Genet 2016; 48: 607-616.

3 Cancer Genome Atlas Research Network. Comprehensive molecular profiling of lung adenocarcinoma. Nature 2014; 511: 543-550.

4 Imielinski M, Berger AH, Hammerman PS, et al. Mapping the hallmarks of lung adenocarcinoma with massively parallel sequencing. Cell 2012; 150: 1107-1120.

$5 \mathrm{Wu} \mathrm{K}$, Zhang X, Li F, et al. Frequent alterations in cytoskeleton remodelling genes in primary and metastatic lung adenocarcinomas. Nat Commun 2015; 6: 10131 .

6 de Bruin EC, McGranahan N, Mitter R, et al. Spatial and temporal diversity in genomic instability processes defines lung cancer evolution. Science 2014; 346: 251-256.

7 de Bruin EC, McGranahan N, Swanton C. Analysis of intratumor heterogeneity unravels lung cancer evolution. Mol Cell Oncol 2015; 2: e985549.

8 Inamura K. Clinicopathological characteristics and mutations driving development of early lung adenocarcinoma: tumor initiation and progression. Int J Mol Sci 2018; 19: E1259.

9 Kobayashi Y, Mitsudomi T, Sakao Y, et al. Genetic features of pulmonary adenocarcinoma presenting with ground-glass nodules: the differences between nodules with and without growth. Ann Oncol 2015; 26: 156-161.

10 Kakinuma R, Noguchi M, Ashizawa K, et al. Natural history of pulmonary subsolid nodules: a prospective multicenter study. J Thorac Oncol 2016; 11: 1012-1028.

11 MacMahon H, Naidich DP, Goo JM, et al. Guidelines for management of incidental pulmonary nodules detected on CT images: from the Fleischner Society 2017. Radiology 2017; 284: 228-243.

12 Sun F, Xi J, Zhan C, et al. Ground glass opacities: imaging, pathology, and gene mutations. J Thorac Cardiovasc Surg 2018; 156: 808-813.

13 Kadara $\mathrm{H}$, Choi $\mathrm{M}$, Zhang $\mathrm{J}$, et al. Whole-exome sequencing and immune profiling of early-stage lung adenocarcinoma with fully annotated clinical follow-up. Ann Oncol 2017; 28: 75-82.

14 Nahar R, Zhai W, Zhang $\mathrm{T}$, et al. Elucidating the genomic architecture of Asian EGFR-mutant lung adenocarcinoma through multi-region exome sequencing. Nat Commun 2018; 9: 216.

15 Izumchenko E, Chang X, Brait M, et al. Targeted sequencing reveals clonal genetic changes in the progression of early lung neoplasms and paired circulating DNA. Nat Commun 2015; 6: 8258.

16 Alexandrov LB, Nik-Zainal S, Wedge DC, et al. Signatures of mutational processes in human cancer. Nature 2013; 500: 415-421.

17 Sanchez-Vega F, Mina M, Armenia J, et al. Oncogenic signaling pathways in the Cancer Genome Atlas. Cell 2018 173: 321-337.

18 Vinayanuwattikun C, Le Calvez-Kelm F, Abedi-Ardekani B, et al. Elucidating genomic characteristics of lung cancer progression from in situ to invasive adenocarcinoma. Sci Rep 2016; 6: 31628.

19 Takamochi K, Takahashi F, Suehara Y, et al. DNA mismatch repair deficiency in surgically resected lung adenocarcinoma: microsatellite instability analysis using the Promega panel. Lung Cancer 2017; 110: 26-31.

20 Li GM. Mechanisms and functions of DNA mismatch repair. Cell Res 2008; 18: 85-98.

21 Ismail IH, Davidson R, Gagné JP, et al. Germline mutations in BAP1 impair its function in DNA double-strand break repair. Cancer Res 2014; 74: 4282-4294.

22 Clynes D, Higgs DR, Gibbons RJ. The chromatin remodeller ATRX: a repeat offender in human disease. Trends Biochem Sci 2013; 38: 461-466.

23 Watanabe R, Ui A, Kanno S, et al. SWI/SNF factors required for cellular resistance to DNA damage include ARID1A and ARID1B and show interdependent protein stability. Cancer Res 2014; 74: 2465-2475.

24 Li R, Li X, Xue R, et al. Early metastasis detected in patients with multifocal pulmonary ground-glass opacities (GGOs). Thorax 2018; 73: 290-292.

25 Zhang C, Zhang J, Xu FP, et al. Genomic landscape and immune microenvironment features of preinvasive and early-invasive lung adenocarcinoma. J Thorac Oncol 2019; 14: 1912-1923.

26 National Comprehensive Cancer Network. NCCN guide-lines for NSCLC. www.nccn.org/professionals/physician_gls Date last accessed: April 2, 2019.

27 Yano M, Yoshida J, Koike T, et al. Survival of 1737 lobectomy-tolerable patients who underwent limited resection for cStage IA non-small-cell lung cancer. Eur J Cardiothorac Surg 2015; 47: 135-142.

28 Wang XW, Chen WF, He WJ, et al. CT features differentiating pre- and minimally invasive from invasive adenocarcinoma appearing as mixed ground-glass nodules: mass is a potential imaging biomarker. Clin Radiol 2018; 73: 549-554 